\title{
Evaluation of a novel handheld point-of-care ultrasound device in an African emergency department
}

\author{
Samuel L. Burleson* ${ }^{*}$, Jonathan F. Swanson, Erin F. Shufflebarger, Douglas W. Wallace, Matthew A. Heimann, \\ James C. Crosby, David C. Pigott, John P. Gullett, Maxwell A. Thompson and Christopher J. Greene
}

\begin{abstract}
Background: Many point-of-care ultrasound devices are now "pocket-sized" or handheld, allowing easy transport during travel and facilitating use in crowded spaces or in austere low-resource settings. Concerns remain about their durability, image quality, and clinical utility in those environments.

Method: Five emergency physicians with training in point-of-care ultrasound employed the Butterfly iQ, a novel handheld ultrasound device, in routine clinical care in a busy, high-acuity African emergency department over a period of 10 weeks. We retrospectively evaluated the performance of the Butterfly iQ from the perspectives of both the clinicians using the device and expert ultrasound faculty reviewing the images.

Results: We found advantages of the Butterfly iQ in a high-acuity African emergency department include its use of a single probe for multiple functions, small size, ease of transport, relatively low cost, and good image quality in most functions. Disadvantages include large probe footprint, lower, though still adequate, cardiac imaging quality, frequent overheating, and reliance on internet-based cloud storage, but these were surmountable. We also report a wide variety of patient presentations, pathology, and procedures to which the device was used.
\end{abstract}

Conclusion: We conclude the Butterfly iQ is an effective, though imperfect, point-of-care ultrasound device in a lowresource emergency setting. We will continue to employ the device in clinical emergency care and teaching in this setting.

Keywords: Point-of-care ultrasound, Handheld, Butterfly iQ, Resource-limited settings, Emergency, Tropical infectious disease

\section{Introduction}

Newly-marketed point-of-care ultrasound (POCUS) devices like the Butterfly iQ (iQ, Butterfly Network, Inc, Guilford, CT, USA) have generated significant excitement over their potential in emergency departments (EDs), critical care units, and resource-limited settings (RLS). Potential benefits of the $\mathrm{iQ}$ include small size, lower cost, integration with the user's mobile phone or tablet, and

\footnotetext{
*Correspondence: slburleson@uabmc.edu

Department of Emergency Medicine, University of Alabama

at Birmingham, Old Hillman Building Suite 251, 619 19th St S,

Birmingham, AL 35249, USA
}

use of silicon-chip based technology obviating the need for multiple transducers. There has been little critical evaluation of the device itself, particularly in RLS. We describe our use of the Butterfly $\mathrm{iQ}$ in routine clinical operations in a busy, high-acuity African ED and review its performance and applicability to RLS.

\section{Personnel and setting}

Five emergency physicians from the United States worked alongside the ED staff of a busy referral hospital in rural east Africa treating medical and surgical patients of all ages over a period of 10 consecutive weeks in the fall of 2019. All physicians have surpassed accepted POCUS 
training guidelines [1]. Two (SLB and JFS) have completed or are enrolled in a Point-of-care Ultrasound in Resource Limited Settings fellowship [2]. Advanced imaging is available, but access is limited by the need for payment prior to testing; in practice, patients often wait hours or days prior to imaging. Patients were selected by clinicians at the bedside if POCUS was indicated as a part of routine clinical care; therefore, informed consent was not required. Patients were scanned with the Butterfly iQ connected to an Apple iPad (iPad 5th Generation, Apple, Cupertino, CA, USA). Images were later reviewed for quality assurance by ultrasound fellowship-trained faculty. Final diagnoses were determined from a combination of the medical record, discussions with inpatient teams, and expert image review.

\section{Results and discussion}

The Butterfly iQ performed well and met clinician needs for a POCUS machine in this single RLS. Its advantages over cart-based machines are magnified, where financial resources, floor space, and reliable power may be scarce. Advantages and disadvantages of the Butterfly iQ are summarized in Table 1.

\section{Advantages}

The combination of flexibility and mobility of a single probe with preset modes replacing multiple transducers is the paramount benefit of the device. We successfully employed the $\mathrm{iQ}$ in a wide variety of scans and patient presentations (see Table 2, Figs. 1, 2, 3) and procedural guidance (see Table 3 ).

Image quality, particularly in the abdominal and musculoskeletal modalities, was excellent, though not generally up to the standards of a cart-based system. With few exceptions (see below), image quality in all modalities was adequate to answer the clinical question.

The software application is user-friendly, allowing rapid alternation between preset scanning modes,

Table 1 Advantages and disadvantages of the butterfly iQ

ADVANTAGES
Single probe replaces multiple traditional transducers, capable of many
scan types
Image quality excellent compared to other handhelds
Low cost
User-friendly app
DISADVANTAGES
Cardiac imaging lower quality than other modes
Relatively frequent overheating
Single probe relatively heavy with large footprint, occasionally compress-
ing small structures

enabling multiple protocols on the same patient with only a few gestures. Some routine calculation functions (such as gestational age) were not available during our experience with the device. We found the increased screen size of an iPad compared to a mobile phone beneficial for most uses.

The cost of the device (approximately US\$2000), plus a required $\$ 420$ annual subscription fee to the cloudbased image storage) places it within reach of some individual clinicians, and many healthcare institutions, even in RLS. Battery life was adequate, usually enough to last an entire 12-h shift on a full charge, though we did not specifically measure continuous scanning time. When necessary, we were able to charge the device from main power on shift. Small, relatively inexpensive, third party solar-powered chargers could also provide additional charging.

\section{Disadvantages}

Echocardiography habitually seemed lower quality than other modes. There was an apparent drop in resolution and frame rate, most noticeably associated with the use of color Doppler. Like other pocket-sized devices, the iQ also lacks spectral Doppler. Despite these limitations, we were able to detect significant cardiovascular pathology. All views were generally obtainable, adequate to guide resuscitation, and answer basic clinical questions pertinent to the RLS [3].

Images are stored on a cloud-based server requiring internet access to upload. Until they are uploaded, the images remain in an "outbox", where they can easily be deleted. Without reliable internet access, many images remained in this "outbox" for the duration of our field work, inhibiting our ability to share or review images.

We encountered several challenges related to the device's hardware. The first was periodic overheating, rendering further scanning impossible until the device cooled. Overheating was not appreciably tied to any specific scanning mode or function. We mitigated this by briefly running water over the waterproof end of the device until cooled. We noticed a small rubber seal loosening near the end of the transducer by the closing of our field experience, without any discernible change in function. We found the cord length $(125 \mathrm{~cm})$ slightly short, especially when performing POCUS-guided procedures. The probe itself weighs $0.7 \mathrm{lb}(0.3 \mathrm{~kg})$, more than twice most other transducers, and its footprint is larger than a phased array probe, which was occasionally problematic when placing ultrasound-guided peripheral IVs and scanning between ribs, respectively. This may have contributed to the decreased quality of some echocardiographic images. 
Table 2 Point-of-care ultrasound findings using Butterfly iQ in African Emergency Department

\begin{tabular}{|c|c|c|c|}
\hline Age/sex & Presentation & US exams & US findings \\
\hline 44 yo $M$ & Afib RVR, Cardiogenic shock & Cardiac & Calcified left atrial thrombus \\
\hline 3 уо M & Abdominal pain, Fussy, Bloody stool & $\mathrm{Gl}$ & Intussusception \\
\hline 28 yo $F$ & Submandibular swelling & MSK & Submandibular abscess \\
\hline 25 yo $M$ & Dyspnea on exertion, $\mathrm{Hx}$ of PCE & Cardiac, Lung & Large PCE without tamponade, Bilateral pleural effusions \\
\hline Young Adult M & Leg pain & MSK & Mid-shaft femur fracture with displacement \\
\hline 23 yo $F$ & Abdominal pain, $\mathrm{Hx}$ of abdominal mass & $\mathrm{Gl}$ & Dermoid cyst (recurrent) \\
\hline 78 yo $M$ & Early satiety, Rectal mass on exam & $\mathrm{Gl}$ & $\begin{array}{l}\text { Novel } d x \text { of diffuse hepatosplenic lesions concerning for } \\
\text { malignancy }\end{array}$ \\
\hline 28 yo $M$ & Necrotic finger & MSK & Abscess vs necrosis \\
\hline $3 \mathrm{moM}$ & Acute respiratory failure & Cardiac, Lung & Novel dx of Atrial Septal Defect \\
\hline 114 yo M & Dyspnea & Cardiac, Lung & Novel dx of HFrEF \\
\hline 13 yo $F$ & Novel Afib and hypoxia, Hx of RHD & Cardiac & $\begin{array}{l}\text { Massive MR, LA dilation, Small PCE (Consistent with } \\
\text { known RHD) }\end{array}$ \\
\hline 14 yo $M$ & Dyspnea, Anasarca on exam, Hx of RHD and malaria & Cardiac & $\begin{array}{l}\text { HFrEF, TR with RA dilation, MR (Consistent with known } \\
\text { RHD) }\end{array}$ \\
\hline 24 yo $M$ & Left flank pain & $\mathrm{Gl}$ & Splenic lesion (Subcapsular hematoma vs Infiltration) \\
\hline 1 yo $M$ & Hypoxia, fever, sepsis & Lung & Bilateral B-lines \\
\hline 1 yo $F$ & Hypoxia, Hx of Ventricular Septal Defect & Cardiac, Lung & $\begin{array}{l}\text { RV Dilation and hypertrophy, Persistent VSD, Bilateral } \\
\text { B-lines }\end{array}$ \\
\hline 74 yo $F$ & Respiratory arrest & Cardiac & Dilated/poorly contractile RV, Full IVC \\
\hline $2 \mathrm{moF}$ & Failure to thrive & CNS & Hydrocephalus \\
\hline Young Adult M & Leg pain & MSK & Mid-shaft tibia fracture with displacement \\
\hline Elderly Adult M & Recent DVT, Dyspnea & Cardiac & Right heart strain \\
\hline 20 yo $F$ & 2-week post-partum, Peritonitis on exam & $\mathrm{Gl}$ & Pelvic free fluid \\
\hline 28 yo $M$ & "Hematemesis" found to be hemoptysis, Hypoxia & Cardiac, Lung, Gl & $\begin{array}{l}\text { Splenic lesion, Bilateral B-lines, Normal LV Ejection Frac- } \\
\text { tion }\end{array}$ \\
\hline Elderly Adult M & Dyspnea, Anasarca on exam, Hypoxia & Cardiac, Lung & $\begin{array}{l}\text { Dilated RA and RV, TR, Bilateral B-lines, Pleural effusions, } \\
\text { Ascites }\end{array}$ \\
\hline 23 yo $F$ & Suicide attempt by drowing, Third trimester pregnancy & $\mathrm{OB}$ & Normal Fetal heart rate and Fetal movement \\
\hline 7 yо $M$ & Abdominal pain, vomiting & $\mathrm{Gl}$ & SBO from worm burden \\
\hline 25 yо M & $\begin{array}{l}\text { Dyspnea and chest pain with near-syncope, Novel Afib } \\
\text { with RVR }\end{array}$ & Cardiac & $\begin{array}{l}\text { Thickened Mitral Valve, Dilated LA, Massive MR (Sus- } \\
\text { pected RHD) }\end{array}$ \\
\hline 5 yо M & Abdominal distention & $\mathrm{Gl}$ & Enlarged bladder with mild hydronephrosis \\
\hline 3 yo $F$ & Constipation, Reports of "worms in stool" & $\mathrm{Gl}$ & SBO, No parasites visualized \\
\hline 85 yo $M$ & RUQ abdominal pain and jaundice & $\mathrm{Gl}$ & Intrahepatic biliary dilation, RUQ Mass \\
\hline 35 yo $F$ & Chest pain, $\mathrm{Hx}$ of Tuberculosis & Lung & Loculated pleural effusion \\
\hline 37 yo $M$ & Abdominal pain, Constipation & $\mathrm{Gl}$ & SBO, Dilated gallbladder \\
\hline 76 yо M & Hypoxia and sepsis & Lung & Subpleural consolidations and B-lines \\
\hline 19 yo $F$ & Hx of tamponade on outpatient echocardiogram & Cardiac & Large PCE, No tamponade \\
\hline 25 yo $M$ & Hypoxia, Novel Afib with RVR & Cardiac & $\begin{array}{l}\text { Massive LA dilation and MR, Thickened anterior mitral } \\
\text { valve leaflet (Suspected RHD) }\end{array}$ \\
\hline
\end{tabular}

Afib, Atrial fibrillation; CNS, Central Nervous System; Dx, Diagnosis; HFrEF, Heart Failure with Reduced Ejection Fraction; Gl, Gastrointestinal/Genitourinary; Hx, History; IVC, Inferior Vena Cava; LA, Left Atrium; MR, Mitral Regurgitation; MSK, Musculoskeletal/Soft tissue; OB, Obstetric; PCE, Pericardial Effusion; RA, Right Atrium; RHD, Rheumatic Heart Disease; RUQ, Right Upper Quadrant; RV, Right Ventricle; RVR, Rapid Ventricular Response; SBO, Small Bowel Obstruction; TR, Tricuspid Regurgitation

\section{Limitations}

These findings represent a retrospective review of the authors' personal experiences with the Butterfly iQ device during routine clinical work in an African ED in an attempt to evaluate its performance in RLS.
Patients were scanned at the discretion of the clinician at the bedside or because of restricted access to other diagnostics, introducing the possibility of selection bias. No patient-oriented outcomes were assessed and no comparisons between devices were available. 


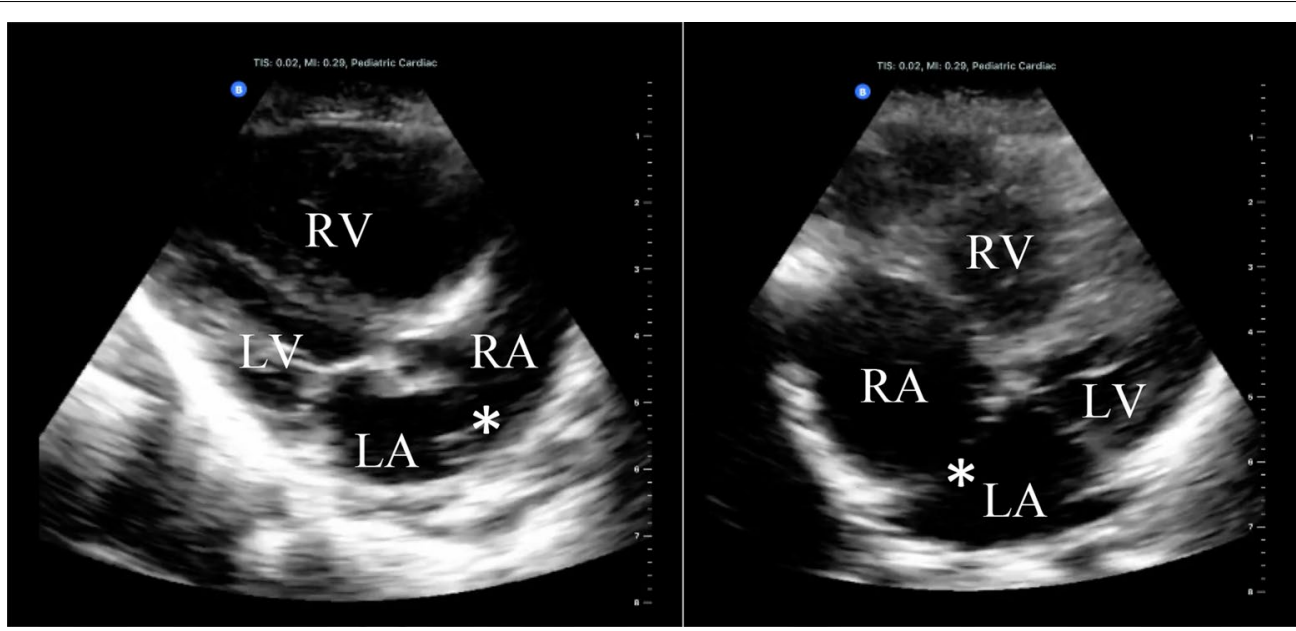

Fig. 1. 3-month-old male presenting with acute respiratory distress. POCUS revealed a dilated right atrium and ventricle with a prominent atrial septal defect, seen in parasternal long view (left) and a slightly modified apical four chamber view (right). LA, Left Atrium, LV, Left Ventricle, RA, Right Atrium, RV, Right Ventricle, * Atrial Septal Defect

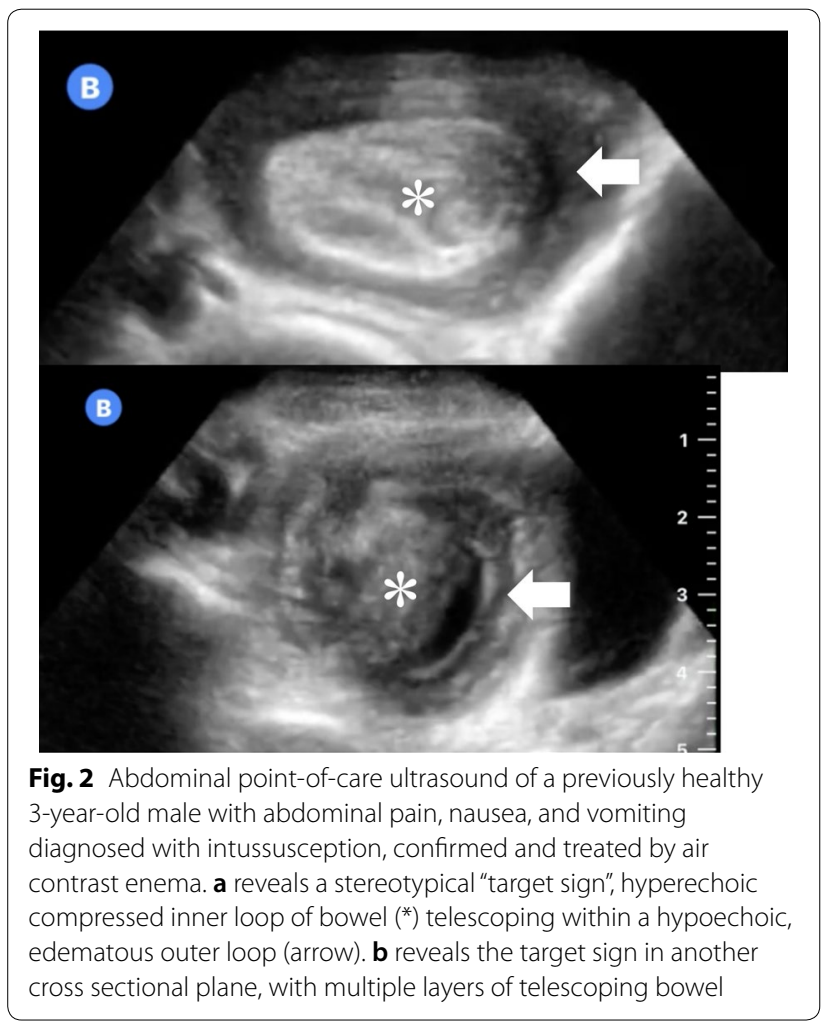

\section{Conclusion}

The Butterfly iQ was employed in a wide variety of patient presentations, scanning indications, and procedural guidance in a busy, high acuity RLS ED. Advantages include its use of a single probe for multiple functions, small size, relatively low cost, and good image quality in most functions. Disadvantages include large probe

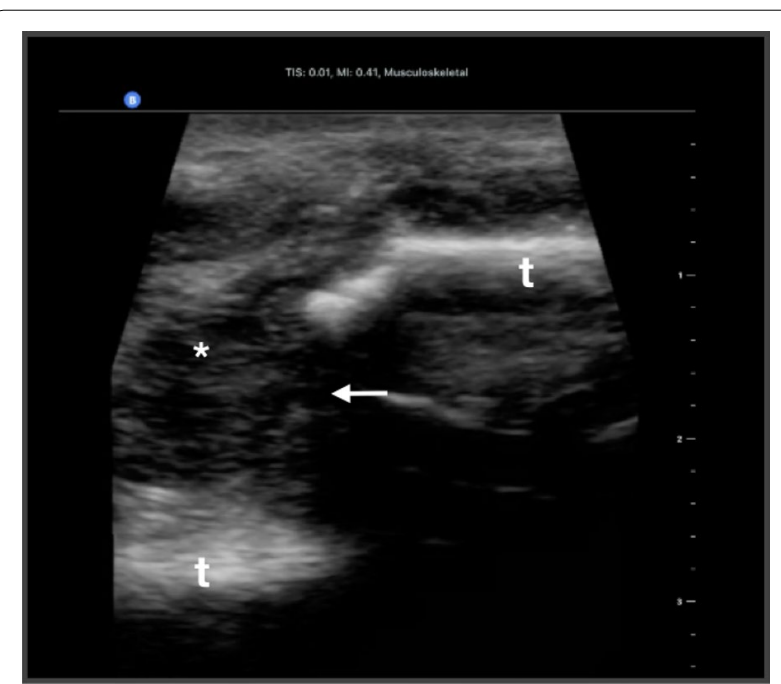

Fig. 3 Musculoskeletal ultrasound of a young male patient with blunt trauma demonstrating two separate sections of tibial cortex $(t)$, with displacement (arrows). Note the associated fracture hematoma visualized $(*)$

\section{Table 3 Procedures guided by Butterfly iQ in an African Emergency Department}

"Easy IJ" - peripheral IV cannula placed in internal jugular vein for shortterm resuscitation [4]

Peripheral IV cannulation

Endotracheal tube placement [5]

Foreign body localization and removal

Paracentesis

Thoracentesis 
footprint, lower, though adequate, cardiac imaging quality, frequent overheating, and reliance on internet-based cloud storage, but these were surmountable. We believe the iQ is an effective POCUS device for emergency care in the RLS and we will continue to employ it for patient care and clinical teaching.

\section{Abbreviations}

ED: Emergency department; POCUS: Point-of-care ultrasound; RLS: Resourcelimited settings.

\section{Acknowledgements}

Not applicable.

\section{Authors' contributions}

SLB - primary and corresponding author, manuscript conceptualization, image acquisition, image interpretation and review, table and figure visualization, editing and revision. JFS—-secondary author, manuscript conceptualization, image acquisition, image interpretation and review, table and figure visualization, editing and revision. EFS—-manuscript conceptualization, image acquisition, image interpretation and review, table and figure visualization, editing and revision. DWW-manuscript conceptualization, image acquisition, image interpretation and review, table and figure visualization, editing and revision. $\mathrm{MAH}$ - manuscript conceptualization, image interpretation and review, table and figure visualization, editing and revision. JCC - manuscript conceptualization, image interpretation and review, table and figure visualization, editing and revision. DCP-manuscript conceptualization, image interpretation and review, editing and revision. JPG - manuscript conceptualization, image interpretation and review, editing and revision. MAT_-manuscript conceptualization, image interpretation and review, editing and revision. CJG — manuscript conceptualization, image acquisition, table and figure visualization, editing and revision. All authors read and approved the final manuscript.

\section{Authors' information}

Authors' information was included under section PERSONELL AND SETTING: Five emergency physicians from the United States worked alongside the ED staff of a busy referral hospital in rural east Africa treating medical and surgical patients of all ages over a period of 10 consecutive weeks in the fall of 2019. All physicians have surpassed accepted ultrasound training guidelines [1]. Two (SLB and JFS) have completed or are enrolled in a Point-of-care Ultrasound in Resource Limited Settings fellowship [2]

\section{Funding}

No funding was received in support of this project.

\section{Availability of data and materials}

All ultrasonographic data is stored on a proprietary cloud-based storage system, as detailed in the text.

\section{Ethics approval and consent to participate}

Not applicable. This manuscript is not a study, but a documentation and evaluation of an-already FDA-approved device in routine clinical care.

\section{Consent for publication}

Not applicable. Consent for publication was not obtained for individual patients. All ultrasound images were obtained as part of routine clinical care, images are de-identified, no specific details or identifying information from the case is presented, and no photos are included.

\section{Competing interests}

No authors have anything to disclose or any financial conflicts of interests. Specifically, no authors have any connection with Butterfly Network, Inc.

Received: 23 June 2020 Accepted: 27 November 2020

Published online: 07 December 2020

\section{References}

1. Accreditation Council for Graduate Medical Education (2016) Program requirements for graduate medical education in emergency medicine. Revised common program requirements. https://urldefense.proof point.com/v2/url? u=https-3A_www.acgme.org_Portals_0_PFAss ets_ProgramRequirements_CPRs-5F2017-2D07-2D01.pdf\&d=DwIGa

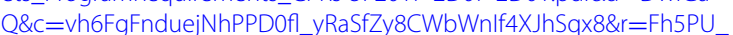
nOJAk3bM1eZddWH6GpaXEY-HwRMRuAL39fctDInX_DZuA9PluyOke3kp $y c \& m=V g M J L 2 j d k U c 8-O i H 1$ iVkdBhkf3nGrnkifYBSKRWdrac\&s=Cjncr AgZvc4a_PEkxXSdMVJ_WmPgbfF10kjzvm5uDR0\&e=. Accessed 27 Nov 2020

2. Burleson SL, Pigott DC, Gullett JP et al (2020) Point-of-care ultrasound in resource-limited settings: the PURLS fellowship. Ultrasound J 12:14

3. Huson MAM, Kaminstein D, Kahn D et al (2019) Cardiac ultrasound in resource-limited settings (CURLS): towards a wider use of basic echo applications in Africa. Ultrasound J 11:34

4. Zitek T, Buby E, Hudson H et al (2018) Ultrasound-guided placement of single-lumen peripheral intravenous catheters in the internal jugular vein. West J Emerg Med 19(5):808-812

5. Gottlieb M, Holladay D, Burns KM et al (2019) Ultrasound for airway management: an evidence-based review for the emergency clinician. Am J Emerg Med. https://doi.org/10.1016/j.ajem.2019/12.019

\section{Publisher's Note}

Springer Nature remains neutral with regard to jurisdictional claims in published maps and institutional affiliations.

\section{Submit your manuscript to a SpringerOpen ${ }^{\circ}$ journal and benefit from:}

- Convenient online submission

- Rigorous peer review

- Open access: articles freely available online

- High visibility within the field

- Retaining the copyright to your article

Submit your next manuscript at $\boldsymbol{\nabla}$ springeropen.com 